\title{
On the influence of interfacial properties to the bending rigidity of layered structures
}

\author{
Shenyou Peng, Yujie Wei* \\ LNM, Institute of Mechanics, Chinese Academy of Sciences, Beijing 100190, PR China
}

\section{A R T I C L E I N F O}

\section{Article history:}

Received 29 January 2016

Received in revised form

24 March 2016

Accepted 5 April 2016

Available online 7 April 2016

Keywords:

Bending stiffness

Interface sliding

Interfacial crack

Multi-layered composite

Bilayer structures

\begin{abstract}
A B S T R A C T
Layered structures are ubiquitous, from one-atom thick layers in two-dimensional materials, to nanoscale lipid bi-layers, and to micro and millimeter thick layers in composites. The mechanical behavior of layered structures heavily depends on the interfacial properties and is of great interest in engineering practice. In this work, we give an analytical solution of the bending rigidity of bilayered structures as a function of the interfacial shear strength. Our results show that while the critical bending stiffness when the interface starts to slide plastically is proportional to the interfacial shear strength, there is a strong nonlinearity between the rigidity and the applied bending after interfacial plastic shearing. We further give semi-analytical solutions to the bending of bilayers when both interfacial shearing and pre-existing crack are present in the interface of rectangular and circular bilayers. The analytical solutions are validated by using finite element simulations. Our analysis suggests that interfacial shearing resistance, interfacial stiffness and preexisting cracks dramatically influence the bending rigidity of bilayers. The results can be utilized to understand the significant stiffness difference in typical biostructures and novel materials, and may also be used for non-destructive detection of interfacial crack in composites when stiffness can be probed through vibration techniques.
\end{abstract}

(c) 2016 Elsevier Ltd. All rights reserved.

\section{Introduction}

In those interface-rich structures, their mechanical properties could be tuned by manipulating the properties of the interfaces. For examples, weak interaction between the lipid-bilayer of cells enables the two layers to slide easily (Boal, 2002; McIntosh and Simon, 2006), as illustrated in Fig. 1a. This mechanism lowers the bending rigidity of the membrane and is beneficial to cell shape-adjustment like fusion or budding of vesicles for intermembrane transportation. In artificial materials, adhesive-bonded components and structures have become a significant part in the aerospace industry and in the automotive industry. Fiber-reinforced metal laminates - hybrid composites consisting of alternating layers of metal sheets and fiber-reinforced epoxy have been adopted for high stiffness, high strength, yet low density (see Fig. 1c). The interfacial properties are deterministic to the overall mechanical behavior of such structures, including stiffness, strength, ductility, damping, durability, and so on (Dugdale, 1960; Hutchinson and Evans, 2000). In view of this, interfacial mechanics has attracted the attention of many engineers and researchers. Many of previous endeavors are to develop physically sound interfacial models (e.g., Xu and Needleman, 1994; Camacho and Ortiz, 1996; Gao and Klein, 1998; Wei, 2014) and apply them

\footnotetext{
* Corresponding author.

E-mail address: yujie_wei@Inm.imech.ac.cn (Y. Wei).
} 


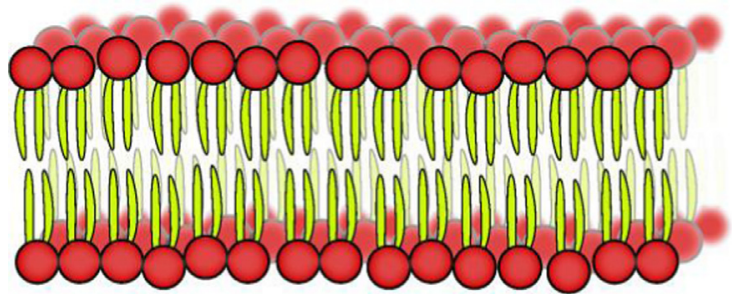

(a)

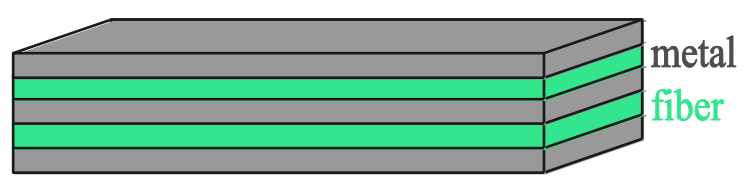

(c)

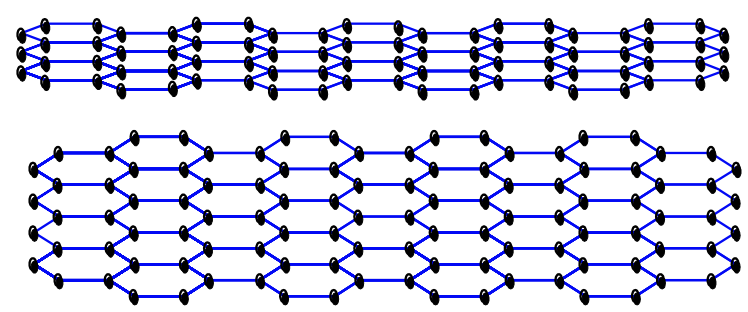

(b)

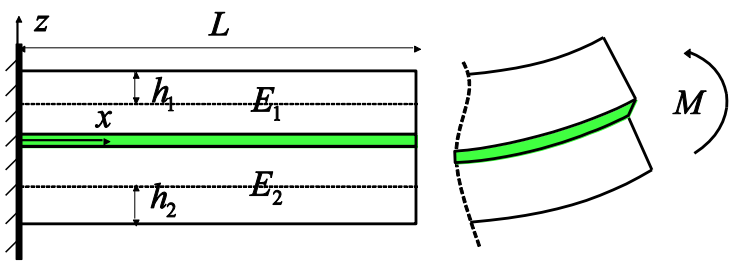

(d)

Fig. 1. Typical layered structures. (a) Lipid-bilayers in cell membranes, the fluidic like interface reduces the bending stiffness of the bilayer and also enhances the mobility of lipids. (b) Bilayer graphene as a result of van der Waals bonding between two monolayers. (c) Composites composed of metal sheets and fiber-reinforced layers. (d) Illustration to show the bending of a bilayered beam: The bending stiffness depends on the interfacial deformation of the layered beam subjected to moment $M$ at its free end when the interface is not rigidly bonded.

to capture the strength and fracture behavior of interface-rich materials and structures. In this work, we focus on developing understanding about how interfacial deformation would influence the stiffness of bilayer structures. The understanding we develop here may not only be utilized to shed light on the mechanical behaviors of broadly seen layered structures in biological systems, but also be used for non-destructive detection of interfacial crack in composites when stiffness can be probed via ultrasonic and acoustic techniques. It could be further used to understand the mechanics of layered materials at nanoscale. For example, it may be applied to illustrate the dramatic difference in bending stiffness of monolayer graphene and bi-layer graphene (Lu et al., 2009; Koening et al., 2011; Shen and Wu, 2012; Wei et al., 2013), with the latter a resultant of binding two monolayer graphenes via van der Waals interactions (see Fig. 1b). It remains unclear how the local electron properties and possible interfacial sliding account for the huge difference in bending stiffness between monolayer graphene and bilayer graphene.

\section{Bilayers with sliding interface}

As a bilayer is the fundamental building block for multilayer materials, we focus our attention on examining the elastic degradation due to the deformation and failure in the interface of a bilayer. The understanding for the bilayer could be directly applied to capture the mechanical behavior of multilayer structures. The mostly cited theory for composite beams was developed by Newmark et al. (1951). The authors applied their model to analysis steel-concrete beams under static load, the layers were treated as Euler-Bernoulli beams and the interface connection was linearly elastic. Since then, extensive models with different assumptions for layered structure has been studied. Adekola (1968) took into account of both slips and uplifts in an interface to look at the deformation of a beam. Gara et al. (2006) extended Adekola's model to handle a wider class of loading and boundary condition. Girhammar and Gopu (1993) and Girhammar and Pan (2007) developed an exact analysis for composite beams by the first order and second order analysis. More recently, effects of shear deformation were investigated by employing Timoshenko beam theory. Schnabl et al. (2007) solved the problem of simply supported bilayer beam with uniformly distributed load. Xu and Wu (2007) obtained analytical solutions for beams subjected to uniformly distributed load with different boundary conditions. More commonly, numerical method are employed to solve the nonlinear problems (Ayoub and Filippou (2000), Salari and Spacone (2001), Cas et al. (2004), Nguyen et al. (2011). It is noted that while previous works laid out the basic strategy to analyze bilayer beams, there is no comprehensive theoretical work to show how the stiffness of layered materials depends on interfacial properties. Analytical and experimental results are available for some special cases like perfectly bonded, free sliding, and a brush interface (Boal, 2002). The purpose of the work is to give an analytical solution on the dependence of the bending rigidity of bilayered structures on the interfacial properties. 


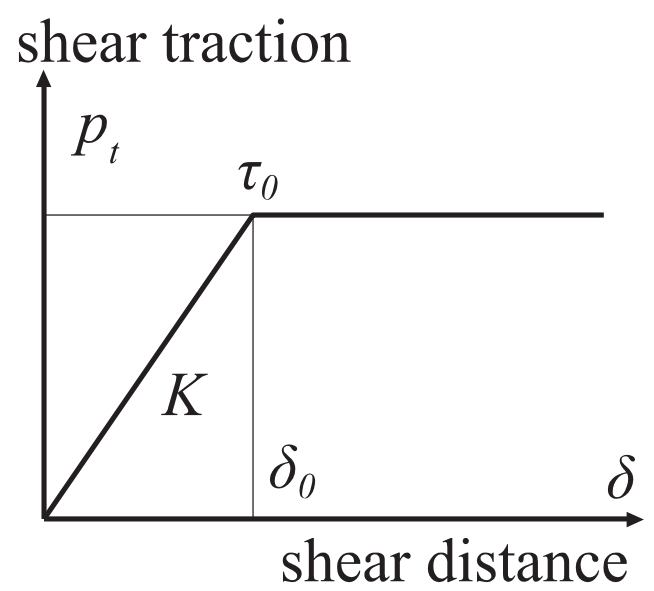

Fig. 2. Shear response of the interface. $\delta[\mathrm{m}]$ denotes the tangential slip between two layers; $p_{t}\left[\mathrm{~N} \mathrm{~m}^{-1}\right]$ is the interfacial shear force per unit length of the beam, as illustrated in Fig. 3. We define $K\left[\mathrm{~N} \mathrm{~m}^{-2}\right]$ as the interfacial stiffness and $\tau_{0}$ as the shear strength.

\subsection{Problem statement}

We consider the bending response of a beam composed of two layers, see the structure shown in Fig. 1d. The two layers could slide with respect to each other when the interfacial shear stress reaches a threshold resistance. For simplicity but without loss of general physics, we consider a slender beam so that it could be treated by the Euler beam theory. To make the problem solvable, we have several assumptions, as listed below.

(1) Deformation in the two layers is linearly elastic, and the Euler beam theory is applicable to the bending of each individual layer.

(2) The interface itself is sufficiently thin (could be as thin as one atomic layer). Therefore, the normal separation in the interface is small and its influence on the mechanical properties of the beam is neglected. The mechanical response to normal deformation is elastic.

(3) As a consequence, the deflections of the two layers are the same: Despite possible interfacial sliding from bending, the interface remains well bound without physical separation. The deflection is a function of the position.

(4) The interfacial shear resistance will reach its plateau once the shearing amount approaches a critical value. No shear failure is considered. The corresponding cohesive zone model to represent the interfacial shearing is illustrated in Fig. 2.

With the assumptions listed above, we are able to formulate the governing equations of the bilayered beam under bending.

\subsection{Governing equations}

With the plane strain condition, we neglect the displacement of the beam in the $y$-axis. The deflection of the beam at $x$ is ascribed as $w=w(x)$. From now on, we use the subscripts ' 1 ' and ' 2 ' to refer to the top and bottom layers, respectively. With the assumptions given in Section 2.1, we may write the displacements of the two layers separately:

$$
u=\left\{\begin{array}{l}
u_{1}(x, z)=u_{10}(x)-\left(z-h_{1}\right) \frac{d w}{d x}, z \geq 0 \\
u_{2}(x, z)=u_{20}(x)-\left(z+h_{2}\right) \frac{d w}{d x}, z \leq 0
\end{array}\right.
$$

with $u_{1}$ and $u_{2}$ referring to the displacements of the top and bottom layers, respectively. The displacements of their respective middle planes are $u_{10}$ and $u_{20}$. Here $h_{1}$ and $h_{2}$ are the heights of the top and the bottom layers, as shown in Fig. $1 \mathrm{~d}$. It is straightforward to write the axial strains $\varepsilon_{x 1}$ and $\varepsilon_{x 2}$ for the respective top and bottom layers as:

$$
\varepsilon_{x}=\left\{\begin{array}{l}
\varepsilon_{x 1}=\frac{\partial u_{1}}{\partial x}=\frac{d u_{10}}{d x}-\left(z-h_{1}\right) \frac{d^{2} w}{d x^{2}}, z \geq 0 \\
\varepsilon_{x 2}=\frac{\partial u_{2}}{\partial x}=\frac{d u_{20}}{d x}-\left(z+h_{2}\right) \frac{d^{2} w}{d x^{2}}, z \leq 0
\end{array}\right.
$$

All other components are approximately zero. Corresponding axial stresses in the top and bottom layers are: 


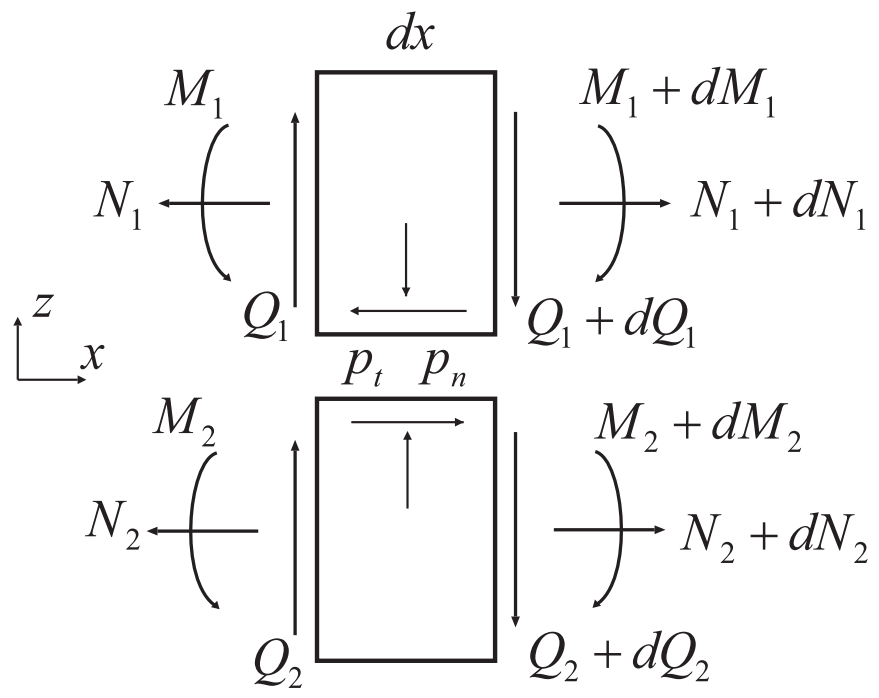

Fig. 3. The free-body diagram of an infinitesimal beam element. Here we consider a free-body of length $d x$ in the bi-layer beam. Parameters $N, M, Q$ stand for axial force, moment, shear stress, respectively, with subscript ' 1 ' for the top layer and ' 2 ' for the bottom layer. The interfacial traction along the tangential and the normal direction are $p_{t}$ and $p_{n}$, respectively.

$$
\sigma_{x}=\left\{\begin{array}{l}
E_{1} \varepsilon_{x 1}, z \geq 0 \\
E_{2} \varepsilon_{x 2}, z \leq 0
\end{array}\right.
$$

where $E_{1}, E_{2}$ are the Young's modulus of the top and bottom layers, respectively. From the axial stress equation, we can derive the axial forces and the bending moments of the two layers as:

$$
\begin{aligned}
& \left\{\begin{array}{l}
N_{1}=b \int_{0}^{2 h_{1}} \sigma_{x} d z=E_{1} A_{1} u_{10}^{\prime} \\
N_{2}=b \int_{-2 h_{2}}^{0} \sigma_{x} d z=E_{2} A_{2} u_{20}^{\prime}
\end{array}\right. \\
& \left\{\begin{array}{l}
M_{1}=b \int_{0}^{2 h_{1}}\left(z-h_{1}\right) \sigma_{x} d z=-E_{1} I_{1} w^{\prime \prime} \\
M_{2}=b \int_{-2 h_{2}}^{0}\left(z+h_{2}\right) \sigma_{x} d z=-E_{2} I_{2} w^{\prime \prime}
\end{array}\right.
\end{aligned}
$$

where $b$ is the out-of-plane thickness of the beam, with $A_{i}=2 b h_{i}(i=1,2)$ representing the cross-section areas of the top and bottom layers, and $I_{i}=\frac{2}{3} b h_{i}^{3}(i=1,2)$ are moments of inertia of each layer. Primes denote differentiation with respect to $x$. Referring to Fig. 3. The equilibrium equations are:

$$
\begin{aligned}
& \left\{\begin{array}{l}
d N_{1}=p_{t} d x \\
d N_{2}=-p_{t} d x
\end{array}\right. \\
& \left\{\begin{array}{l}
d Q_{1}=-p_{n} d x \\
d Q_{2}=p_{n} d x
\end{array}\right. \\
& d M_{i}=-Q_{i} d x-p_{t} h_{i} d x(i=1,2)
\end{aligned}
$$

Now, we can formulate the relations between interfacial shear force $p_{t}$ and displacements of the beams $u_{10}, u_{20}$ and $w$. Combining above Eqs. (4)-(8) and eliminating the interfacial normal force $p_{n}$, we have

$$
\begin{gathered}
E_{1} A_{1} u_{10}^{\prime \prime}=p_{t} \\
E_{2} A_{2} u_{20}^{\prime \prime}=-p_{t} \\
\left(E_{1} I_{1}+E_{2} I_{2}\right) w^{\prime \prime \prime}=\left(h_{1}+h_{2}\right) p_{t}
\end{gathered}
$$

We now consider the displacement jump across the interface:

$$
\delta=u_{1}(x, 0)-u_{2}(x, 0)=u_{10}-u_{20}+\left(h_{1}+h_{2}\right) w^{\prime}
$$

Differentiating Eq. (10) by twice and then combining it with Eq. (9), we have 
$\delta^{\prime \prime}=\left(\frac{1}{E_{1} A_{1}}+\frac{1}{E_{2} A_{2}}+\frac{\left(h_{1}+h_{2}\right)^{2}}{E_{1} I_{1}+E_{2} I_{2}}\right) p_{t}$

As shown in Fig. 2, we use a simple constitute equation to connect the interface shear force $p_{t}$ and the interface slip $\delta$ :

$$
p_{t}=\left\{\begin{array}{l}
K \delta, \text { elastic } \\
\tau_{0}, \text { yielding }
\end{array}\right.
$$

For a cantilever beam under pure bending, we know the boundary conditions as:

$$
\begin{gathered}
\delta(0)=0 \\
w(0)=w^{\prime}(0)=0 \\
\left.\left(M_{1}+M_{2}\right)\right|_{x=L}=-M \\
\left.N_{1}\right|_{x=L}=\left.N_{2}\right|_{x=L}=0
\end{gathered}
$$

Substituting Eq. (5) into the third line of Eq. (13), we obtain the boundary conditions for the deflection:

$$
\begin{gathered}
w^{\prime \prime}(\mathrm{L})=\frac{1}{E_{1} I_{1}+E_{2} I_{2}} M \\
w(0)=w^{\prime}(0)=0
\end{gathered}
$$

Differentiating Eq. (10) and combining the result with Eqs. (4), (5) and (13), we may write the boundary condition in Eq. (13) in the following form:

$$
\begin{gathered}
\delta(0)=0 \\
\delta^{\prime}(L)=\frac{h_{1}+h_{2}}{E_{1} I_{1}+E_{2} I_{2}} M
\end{gathered}
$$

For convenience, we rewrite Eqs. (11) and (15) as follows:

$$
K \delta^{\prime \prime}=A^{2} p_{t}
$$

$$
\begin{gathered}
\delta(0)=0 \\
K \delta^{\prime}(L)=B M
\end{gathered}
$$

Here $A$ and $B$ are defined as:

$$
A^{2}=K\left(\frac{1}{E_{1} A_{1}}+\frac{1}{E_{2} A_{2}}+\frac{\left(h_{1}+h_{2}\right)^{2}}{E_{1} I_{1}+E_{2} I_{2}}\right), B=K \frac{h_{1}+h_{2}}{E_{1} I_{1}+E_{2} I_{2}} .
$$

\subsection{The analytical solution}

From the governing equations obtained in Section 2.2, we could proceed to derive the analytical solutions for the beam under pure bending. We first solve for $\delta(x)$ and $p_{t}(x)$ using Eqs. (12) and (16). The general solutions of $\delta(x)$ and $p_{t}(x)$ are:

$$
\begin{aligned}
& K \delta(x)=\left\{\begin{array}{l}
C_{0} \cosh A x+C_{1} \sinh A x, \text { elastic } \\
\frac{1}{2} A^{2} \tau_{0} x^{2}+C_{2} x+C_{3} \text {,yielding }
\end{array}\right. \\
& p_{t}(x)=\left\{\begin{array}{l}
C_{0} \cosh A x+C_{1} \sinh A x \text {,elastic } \\
\tau_{0}, \text { yielding }
\end{array}\right.
\end{aligned}
$$

The coefficients in the above equations can be determined using the boundary conditions given in Eq. (17); hence the exact solutions can be obtained. Inserting this result into the third of Eq. (9) and applying the boundary condition in Eq. (14), we obtain the deflection of the beam. By definition, we have the bending stiffness $S_{e}=M / \theta$, here the moment $M$ is exerted at the free end of the beam, and $\theta$ is the rotation at the end. We approach the solution by considering separately two states of the interface: without interfacial plastic sliding and with plastic sliding after the shear stress reaches its plateau.

\subsubsection{Elastic interfacial sliding}

We first consider the situation when the interface of the beam deforms elastically. There is no interfacial yielding. We have:

$$
K \delta(x)=p_{t}(x)=c_{0} \cosh A x+c_{1} \sinh A x
$$


for $x \in[0, L]$. Applying the initial conditions in Eq. (17), we obtain:

$$
\begin{gathered}
c_{0}=0 \\
c_{1}=\frac{B M}{A \cosh A L}
\end{gathered}
$$

Now we have the interfacial traction $p_{t}$. Substituting the result into the third line of Eq. (9), we have

$$
S w^{\prime \prime \prime}=\frac{B\left(h_{1}+h_{2}\right)}{A} \frac{\sinh A x}{\cosh A L} M
$$

where $S=E_{1} I_{1}+E_{2} I_{2}$. We then apply the boundary conditions in Eq. (14) and obtain:

$$
S w=\left(\frac{1-k}{2} x^{2}+\frac{k}{A^{2}} \frac{\cosh A x-1}{\cosh A L}\right) M
$$

where

$$
k=\frac{B\left(h_{1}+h_{2}\right)}{A^{2}}=\frac{\left(h_{1}+h_{2}\right)^{2}}{E_{1} I_{1}+E_{2} I_{2}} /\left(\frac{1}{E_{1} A_{1}}+\frac{1}{E_{2} A_{2}}+\frac{\left(h_{1}+h_{2}\right)^{2}}{E_{1} I_{1}+E_{2} I_{2}}\right)
$$

Corresponding rotation at the free-end of the beam is obtained from the deflection curve:

$$
\theta=\frac{M}{S}\left[(1-k) L+\frac{k}{A} \tanh A L\right]
$$

Now we get the bending rigidity defined previously:

$$
S_{e}=\frac{M}{\theta}=\frac{S}{(1-k) L+\frac{k}{A} \tanh A L}=\frac{S / L}{(1-k)+\frac{k}{\beta} \tanh \beta}
$$

For simplicity, we also adopt the definition suggested by Girhammar and Pan (2007) and use

$$
\beta=A L=\left[K\left(\frac{1}{E_{1} A_{1}}+\frac{1}{E_{2} A_{2}}+\frac{\left(h_{1}+h_{2}\right)^{2}}{E_{1} I_{1}+E_{2} I_{2}}\right)\right]^{\frac{1}{2}} L
$$

where $\beta$ is the so-called composite action parameter. It quantifies the impact of interfacial rigidity on the beam rigidity.

\subsubsection{Elastic-plastic interfacial sliding}

If we continue to increase the bending moment, the shear force in the interface increases and may reach the critical shear resistance of the interface. As a result, part of the interface may experience perceivable amount of plastic shearing while the interface remains bound. We now consider this circumstance and solve the differential equation for deflection when $M>M_{0} \cdot M_{0}$ is the critical bending moment. We made the assumption that crack will initiate from the free end where stress concentration occurs. Since plastic sliding will start from the free end and move toward the clamped end, we let the point separating the elastic and plastic sliding at $x=l_{0}$. From $l_{0}$ to $L$, the shear force in the interface would be its shearing resistance. Hence we have

$$
\begin{aligned}
& K \delta(x)=\left\{\begin{array}{l}
c_{0} \cosh A x+c_{1} \sinh A x, 0<x<l_{0} \\
\frac{1}{2} A^{2} \tau_{0} x^{2}+c_{2} x+c_{3}, l_{0}<x<L
\end{array}\right. \\
& p_{t}(x)=\left\{\begin{array}{l}
c_{0} \cosh A x+c_{1} \sinh A x, 0<x<l_{0} \\
\tau_{0}, l_{0}<x<L
\end{array}\right.
\end{aligned}
$$

Combining with the boundary condition in Eq. (17) and the continuous conditions of $\delta(x)$ and $\delta^{\prime}(x)$ at the point $x=l_{0}$, we obtain the two unknowns in Eq. (30):

$$
c_{1}=\frac{B M-A^{2} \tau_{0}\left(L-l_{0}\right)}{A \cosh A l_{0}}
$$

From the assumption, we have the following condition at point $x=l_{0}$ :

$$
c_{0} \cosh A l_{0}+c_{1} \sinh A l_{0}=\tau_{0}
$$

Applying this stress boundary, we have 


$$
M=\frac{A}{B} \tau_{0} \operatorname{coth} A l_{0}+\frac{A^{2}}{B} \tau_{0}\left(L-l_{0}\right)
$$

So now we obtain the interfacial force $p_{t}$. That is, the relationship between $p_{t}$ and bending moment $M$ is built indirectly via the condition at $l_{0}$. The corresponding critical bending moment which triggers the yielding of the interface, by setting $l_{0}=L$, is given as:

$$
M_{0}=\frac{A}{B} \tau_{0} \operatorname{coth} A L
$$

Since $p_{t}$ is obtained, inputting $p_{t}$ into the third of Eq. (9) and using the boundary condition in Eq. (14), we get the deflection. In order to reduce the unknowns while solving the third order partial differential Eq. (9), we rewrite it into a second order differential equation as:

$$
\left\{\begin{array}{l}
S w^{\prime \prime}=M-\int_{x}^{L}\left(h_{1}+h_{2}\right) p_{t} d x \\
w(0)=w^{\prime}(0)=0
\end{array}\right.
$$

From Eq. (35), the deflection of the beam is:

$$
\left\{\begin{array}{l}
S w=\frac{1-k}{2} M x^{2}+\frac{\left(h_{1}+h_{2}\right) \tau_{0}}{A^{3} \sinh A l_{0}} \cosh A x+\theta_{1} x+d_{1}, 0<x<l_{0} \\
S w=\frac{1}{2}\left[M-\left(h_{1}+h_{2}\right) \tau_{0} L\right] x^{2}+\frac{1}{6}\left(h_{1}+h_{2}\right) \tau_{0} x^{3}+\theta_{2} x+d_{2}, l_{0}<x<L
\end{array}\right.
$$

Using the boundary condition for the deflection $w(0)=w^{\prime}(0)=0$ and the continuous conditions for $w(x)$ and $w^{\prime}(x)$ at point $x=l_{0}$, we could obtain the deflection curve by iteration. Hence the bending rigidity of the beam could be deduced:

$$
S_{e}=\frac{M}{\theta}=\frac{M}{w^{\prime}(L)}=S_{e}\left(K, \tau_{0}, M\right)
$$

It is not surprise to see that the stiffness of the beam is now a function of the applied bending moment, the shear modulus of the interface, and the critical shear resistance of the interface.

\section{A bilayer with interfacial crack and sliding}

In the previous section, we formulated the equations of bending stiffness as a function of interfacial shearing resistance. In that scenario, there are no cracks in the interface, which is suitable for problems like lipid bilayers with weak resistance to relative sliding. Now we extend the analysis to consider a bilayer with pre-existing interfacial crack and possible interfacial sliding. Problems of these kinds are broadly seen in composite materials.

\subsection{Problem description}

We consider the same type of bilayers but with a pre-existing crack in the middle of the interface, as illustrated in Fig. 4. We are interested in how the pre-existing crack would degrade the bending rigidity of the bilayer. The general solution of $\delta(x)$ and $p_{t}(x)$ becomes:

$$
K \delta(x)=\left\{\begin{array}{l}
C_{0} \cosh A x+C_{1} \text { sinh } A x, \text { elastic region } \\
C_{2} x+C_{3}, \text { cracked region } \\
\frac{1}{2} A^{2} \tau_{0} x^{2}+C_{4} x+C_{5}, \text { yield region }
\end{array}\right.
$$

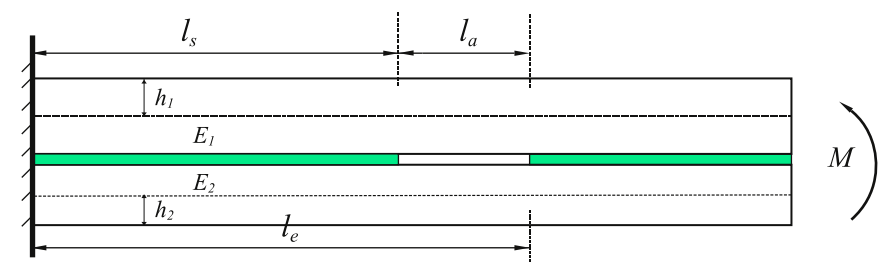

Fig. 4. Illustration for the bending of layered structures with pre-existing interfacial crack. The white region in the interface is the initial crack. 
$p_{t}(x)=\left\{\begin{array}{l}C_{0} \cosh A x+C_{1} \sinh A x, \text { elastic region } \\ 0, \text { cracked region } \\ \tau_{0}, \text { yield region }\end{array}\right.$

Then we use Eq. (35) to deduce the deflection and hence the bending rigidity.

\subsection{Semi-analytical solutions}

Following the deformation process, the interfacial shear stress increases with increasing bending moment. To some level, the bending moment will be high enough to trigger interface yield. The yield region may growth in response to further increasing in bending moment. The whole process can be divided into four steps and we supply corresponding semianalytical solutions for each case.

\subsubsection{Bending before interfacial elastic sliding}

As the bending moment increases, the interface first experiences an elastic stage when no yield occurs in any portion of interface, i.e. $\left(M<M_{1}\right) \cdot M_{i}(i=1,2,3)$ are the respective critical values for those four steps. We first write the equations for interfacial shear stress. From the general solution to Eq. (11), we have

$$
\begin{gathered}
K \delta(x)=\left\{\begin{array}{l}
c_{0} \cosh A x+c_{1} \sinh A x, 0<x<l_{s} \\
c_{2} x+c_{3}, l_{s}<x<l_{e} \\
c_{4} \cosh A x+c_{5} \sinh A x, l_{e}<x<L
\end{array}\right. \\
p_{t}(x)=\left\{\begin{array}{l}
c_{0} \cosh A x+c_{1} \sinh A x, 0<x<l_{s} \\
0, l_{s}<x<l_{e} \\
c_{4} \cosh A x+c_{5} \sinh A x, l_{e}<x<L
\end{array}\right.
\end{gathered}
$$

where $l_{s}$ and $l_{a}$ are the geometry parameters shown in Fig. $4, l_{e}=l_{s}+l_{a}$. With the boundary conditions in Eq. (17) and the continuous conditions of $\delta(x)$ and $\delta^{\prime}(x)$ at the points $x=l_{s}$ and $x=l_{e}$, we have

$$
\left\{\begin{array}{l}
c_{4}=c_{41} c_{1}, c_{5}=c_{51} c_{1}, c_{0}=0 \\
c_{1}=\frac{B M}{A\left(c_{41} \sinh A L+c_{51} \cosh A L\right)} \\
c_{41}=A l_{a} \cosh A l_{s} \cosh A l_{e}+\sinh A l_{s} \cosh A l_{e}-\cosh A l_{s} \sinh A l_{e} \\
c_{51}=-\left(A l_{a} \cosh A l_{s} \sinh A l_{e}+\sinh A l_{s} \sinh A l_{e}-\cosh A l_{s} \cosh A l_{e}\right)
\end{array}\right.
$$

Since no yield occurs, $p_{t}$ satisfies $p_{t}(L)<\tau_{0}$. That is, $c_{4} \cosh A L+c_{5} \sinh A L<\tau_{0}$. This condition can be further shown to be:

$$
M<\frac{A}{B} \tau_{0} \frac{c_{41} \sinh A L+c_{51} \cosh A L}{c_{41} \cosh A L+c_{51} \sinh A L}=M_{1}
$$

Now we proceed to solve the deflection equation $w(x)$. Inputting Eq. (41) into Eq. (35), we have

$$
\left\{\begin{array}{l}
S w=\frac{1-k}{2} M x^{2}+\frac{h_{1}+h_{2}}{A^{3}} c_{1} \cosh A x+\theta_{1} x+d_{1}, 0<x<l_{s} \\
S w=\frac{1-k}{2} M x^{2}+\frac{h_{1}+h_{2}}{A}\left(c_{4} \sinh A l_{e}+c_{5} \cosh A l_{e}\right) \frac{x^{2}}{2}+\theta_{2} x+d_{2}, l_{s}<x<l_{e} \\
S w=\frac{1-k}{2} M x^{2}+\frac{h_{1}+h_{2}}{A^{3}}\left(c_{4} \sinh A x+c_{5} \cosh A x\right)+\theta_{3} x+d_{3}, l_{e}<x<L
\end{array}\right.
$$

Combining with the boundary conditions $w(0)=w^{\prime}(0)=0$ and the continuous conditions for $w(x)$ and $w^{\prime}(x)$, we can solve for the deflection equation by numeric method.

\subsubsection{Bending after partial interfacial plastic sliding}

With further increase in the bending moment, interfacial shear stress increases and may trigger part of the interface to yield. We consider the situation that the bending moment satisfies $M_{1}<M<M_{2}$, so that part of interface on right-hand side of the pre-existing crack yields. Let the yield occurs within $l_{0}$ to $L$. Now the general solution for shearing displacement and shear stress are given as 


$$
\begin{gathered}
K \delta(x)=\left\{\begin{array}{l}
c_{0} \cosh A x+c_{1} \sinh A x, 0<x<l_{s} \\
c_{2} x+c_{3}, l_{s}<x<l_{e} \\
c_{4} \cosh A x+c_{5} \sinh A x, l_{e}<x<l_{0} \\
\frac{1}{2} A^{2} \tau_{0} x^{2}+C_{6} x+C_{7}, l_{0}<x<L
\end{array}\right. \\
p_{t}(x)=\left\{\begin{array}{lr}
c_{0} \cosh A x+c_{1} \sinh A x, & 0<x<l_{s} \\
0, & l_{s}<x<l_{e} \\
c_{4} \cosh A x+c_{5} \sinh A x, & l_{e}<x<l_{0} \\
\tau_{0}, & l_{0}<x<L
\end{array}\right.
\end{gathered}
$$

with

$$
\begin{gathered}
c_{4}=c_{41} c_{1}, c_{5}=c_{51} c_{1}, c_{0}=0 \\
c_{1}=\frac{B M-A^{2} \tau_{0}\left(L-l_{0}\right)}{A\left(c_{41} \sinh A l_{0}+c_{51} \cosh A l_{0}\right)}
\end{gathered}
$$

Here we only need to solve the unknowns in the expression of $p_{t}(x)$. At $x=l_{0}$, we have $c_{4} \cosh A l_{0}+c_{5} \sinh A l_{0}=\tau_{0}$. This condition suggests that the bending moment satisfies:

$$
M=\frac{A}{B} \tau_{0} \frac{c_{41} \sinh A l_{0}+c_{51} \cosh A l_{0}}{c_{41} \cosh A l_{0}+c_{51} \sinh A l_{0}}+\frac{A^{2}}{B} \tau_{0}\left(L-l_{0}\right)
$$

It is also less than the critical bending moment which triggers the yield of the whole interface in the right-hand-side of the crack. That indicates:

$$
M_{2}=\frac{A}{B} \tau_{0} \frac{c_{41} \sinh A l_{e}+c_{51} \cosh A l_{e}}{c_{41} \cosh A l_{e}+c_{51} \sinh A l_{e}}+\frac{A^{2}}{B} \tau_{0}\left(L-l_{e}\right)
$$

Corresponding deflection equations can be given as:

$$
\left\{\begin{array}{l}
S w=\frac{1-k}{2} M x^{2}+\frac{h_{1}+h_{2}}{A^{3}} c_{1} \cosh A x+\theta_{1} x+d_{1}, 0<x<l_{s} \\
S w=\frac{1-k}{2} M x^{2}+\frac{h_{1}+h_{2}}{A}\left(c_{4} \sinh A l_{e}+c_{5} \cosh A l_{e}\right) \frac{x^{2}}{2}+\theta_{2} x+d_{2}, l_{s}<x<l_{e} \\
S w=\frac{1-k}{2} M x^{2}+\frac{h_{1}+h_{2}}{A^{3}}\left(c_{4} \sinh A x+c_{5} \cosh A x\right)+\theta_{3} x+d_{3}, l_{e}<x<l_{0} \\
S w=\frac{1}{2}\left[M-\left(h_{1}+h_{2}\right) \tau_{0} L\right] x^{2}+\frac{1}{6}\left(h_{1}+h_{2}\right) \tau_{0} x^{3}+\theta_{4} x+d_{4}, l_{0}<x<L
\end{array}\right.
$$

With known information for the bending moment and the resultant deflection, it is straightforward to deduce the bending rigidity of the bilayer beam in a particular loading step.

\subsubsection{Bending after complete plastic sliding in one part}

No surprisingly, the yield zone will grow in response to further increasing bending moment when it falls in the regime of $M_{2}<M<M_{3}$. Now the whole surface on the right-hand side of the crack yields, but no yield happens in the interface on the left-hand side of the crack. Corresponding interfacial shear stress in the bilayer is given as:

$$
p_{t}(x)=\left\{\begin{array}{l}
c_{0} \cosh A x+c_{1} \sinh A x, 0<x<l_{s} \\
0, l_{s}<x<l_{e} \\
\tau_{0}, l_{e}<x<L
\end{array}\right.
$$

There are only two unknowns, which could be determined from boundary conditions with the same method employed previously, thus

$$
c_{0}=0, c_{1}=\frac{B M-A^{2} \tau_{0}\left(L-l_{e}\right)}{A \cosh A l_{s}}
$$

Since we require $c_{4} \cosh A l_{s}+c_{5} \sinh A l_{s}<\tau_{0}$, it turns out the bending moment satisfies:

$$
M<\frac{A}{B} \tau_{0} \operatorname{coth} A l_{s}+\frac{A^{2}}{B} \tau_{0}\left(L-l_{e}\right)=M_{3}
$$


Similarly, now the deflection is obtained as

$$
\left\{\begin{array}{l}
S w=\frac{1-k}{2} M x^{2}+\frac{h_{1}+h_{2}}{A^{3}} c_{1} \cosh A x+\theta_{1} x+d_{1}, 0<x<l_{s} \\
S w=\frac{1}{2}\left[M-\left(h_{1}+h_{2}\right) \tau_{0}\left(L-l_{e}\right)\right] x^{2}+\theta_{2} x+d_{2}, l_{s}<x<l_{e} \\
S w=\frac{1}{2}\left[M-\left(h_{1}+h_{2}\right) \tau_{0} L\right] x^{2}+\frac{1}{6}\left(h_{1}+h_{2}\right) \tau_{0} x^{3}+\theta_{3} x+d_{3}, l_{e}<x<L
\end{array}\right.
$$

From which the bending rigidity could be determined.

\subsubsection{Bending after plastic sliding in two parts}

After the whole right-hand side of the interface yields, we will experience a nonlinear increase of deflection as the bending moment increases to the critical level when the left-hand side of the interface starts to yield. That is, $M>M_{3}$. The shear stress in this circumstance is described as:

$$
p_{t}(x)=\left\{\begin{array}{l}
c_{0} \cosh A x+c_{1} \sinh A x, 0<x<l_{0} \\
0, l_{s}<x<l_{e} \\
\tau_{0}, l_{0}<x<l_{s} \text { and } l_{e}<x<L
\end{array}\right.
$$

with

$$
c_{0}=0, c_{1}=\frac{B M-A^{2} \tau_{0}\left(L-l_{a}-l_{0}\right)}{A \cosh A l_{0}}
$$

At $l_{0}$, we have $c_{0} \cosh A l_{0}+c_{1} \sinh A l_{0}=\tau_{0}$. Writing this condition in terms of the bending moment, we have

$$
M=\frac{A}{B} \tau_{0} \operatorname{coth} A l_{0}+\frac{A^{2}}{B} \tau_{0}\left(L-l_{a}-l_{0}\right)
$$

The deflection is hence given as:

$$
\left\{\begin{array}{l}
S w=\frac{1-k}{2} M x^{2}+\frac{h_{1}+h_{2}}{A^{3}} c_{1} \cosh A x+\theta_{1} x+d_{1}, 0<x<l_{0} \\
S w=\frac{1}{2}\left[M-\left(h_{1}+h_{2}\right) \tau_{0}\left(L-l_{a}\right)\right] x^{2}+\frac{1}{6}\left(h_{1}+h_{2}\right) \tau_{0} x^{3}+\theta_{2} x+d_{2}, l_{0}<x<l_{s} \\
S w=\frac{1}{2}\left[M-\left(h_{1}+h_{2}\right) \tau_{0}\left(L-l_{e}\right)\right] x^{2}+\theta_{3} x+d_{3}, l_{s}<x<l_{e} \\
S w=\frac{1}{2}\left[M-\left(h_{1}+h_{2}\right) \tau_{0} L\right] x^{2}+\frac{1}{6}\left(h_{1}+h_{2}\right) \tau_{0} x^{3}+\theta_{4} x+d_{4}, l_{e}<x<L
\end{array}\right.
$$

And the bending rigidity could be obtained following the same procedures defined above. In conclusion, the bending rigidity of the bilayer beam with a pre-existing crack and considering the interfacial slip can be given as:

$$
S_{e}=S_{e}\left(K, \tau_{0}, l_{s}\left(l_{e}\right), l_{a}, M\right)
$$

In the next section, we will present applications of the theories to typical bilayers and examine the predictability of the theories against numerical simulation results.

\section{Numerical verification}

In the previous two sections, we have shown theoretically how both shearing and pre-existing crack in the interface of a bilayer beam would influence the bending rigidity of the beam. As there involve several assumptions when we derive the analytical formula for both cases, we choose finite element simulations to further examine the validity of those theoretical analysis.

\subsection{Simulation details}

We consider a bilayer beam with its left end clamped to a rigid wall. We apply bending moment at the free end of the beam. The thicknesses of the top and bottom layers are $h_{1}=0.5 \mathrm{~cm}$ and $h_{2}=0.25 \mathrm{~cm}$, respectively. Plane stress boundary condition is assumed, and we take the out-of-plane thickness of the beam to be $b=1 \mathrm{~cm}$. The length of the beam is assumed to be $L=10 \mathrm{~cm}$. We assume the two layers deform elastically. The Young's modulus of the top layer is $E_{1}=60 \mathrm{GPa}$ respectively; that of the bottom layer is $E_{2}=80 \mathrm{GPa}$. For the interfacial response, we consider a shear traction-separation law shown in 
Table 1

Geometry parameters and material parameters for two types of bilayers: Case A (Two layers with different properties) and case B (Two same layers). Here $S_{0}=\left(E_{1} I_{1}+E_{2} I_{2}\right) / L$ denotes the rigidity of unbonded beam, and $M_{0}$ denotes the critical yielding bending moment with a particular interfacial property of $K=0.8 \mathrm{Gpa}$ and $\tau_{0}=0.5 \mathrm{Mpa} \cdot \mathrm{m}$. We use $S_{0}, M_{0}$ to normalize the bending rigidity and applied bending moment for all figures. Indeed, as $b$ is the out-of-plane dimension, it could be any number in the plane-strain problem. For simplicity, we take $b=1 \mathrm{~cm}$ so that all other parameters will have their normal dimensions.

\begin{tabular}{ll}
\hline Case A & Case B \\
\hline$E_{1}=60 \mathrm{GPa}, h_{1}=0.50 \mathrm{~cm}$ & $E_{1}=80 \mathrm{GPa}, h_{1}=0.25 \mathrm{~cm}$ \\
$E_{2}=80 \mathrm{GPa}, h_{2}=0.25 \mathrm{~cm}$ & $E_{2}=80 \mathrm{GPa}, h_{2}=0.25 \mathrm{~cm}$ \\
$b=1 \mathrm{~cm}$ & $b=1 \mathrm{~cm}$ \\
$L=10 \mathrm{~cm}$ & $L=10 \mathrm{~cm}$ \\
$S_{0}=\left(E_{1} I_{1}+E_{2} I_{2}\right) / L=583 \mathrm{~N} \cdot \mathrm{m}$ & $S_{0}=\left(E_{1} I_{1}+E_{2} I_{2}\right) / L=167 \mathrm{~N} \cdot \mathrm{m}$ \\
$M_{0}=162 \mathrm{~N} \cdot \mathrm{m}$ & $M_{0}=83.4 N \cdot \mathrm{m}$ \\
\hline
\end{tabular}

Fig. 2. For comparison, we also present theoretical predictions and simulation results for the bilayer beam of identical layers: $h_{1}=h_{2}=0.25 \mathrm{~cm}, L=10 \mathrm{~cm}$, and $E_{1}=E_{2}=80 \mathrm{GPa}$. All used parameters are given in Table 1 .

We checked the element type and its influence on convergence. It turns out that the two-dimensional element type SPS8R for the solids and the COH2D4 cohesive element in the interface work well for our calculations. Ten elements along the beam thickness for each layer is sufficient for convergence. Because of the cohesive element we used in Abaqus only gives the stress and strain, the properties of the interface we set need to be transformed into the properties we used in the previous sections by taking into account the interfacial geometry. To maintain consistence, the parameters we investigated are given following the definition in Section 2 (see Fig. 2).

\subsection{The crack-free case}

From the previous discussion we know that, for a particular bilayered structure, both interfacial stiffness $(K)$ and strength $\left(\tau_{0}\right)$ contribute to its bending rigidity, i.e. $S_{e}=S_{e}\left(K, \tau_{0}, M\right)$. For convenience, we first concentrate on the influence of interfacial stiffness with a fixed interfacial shear resistance $\tau_{0}=0.5 \mathrm{Mpa} \mathrm{m}$, hence $S_{e}=S_{e}(K, M)$. The analytical and simulation results show in Fig. 5a. The analytical result consists of two parts: elastic deformation and post-elastic deformation. The elastic portion is obtained by solving Eq. (27); for the post-elastic portion, we solve Eqs. (36) and (37) to get the relation for $S_{e}=S_{e}\left(K, \tau_{0}, M, l_{0}\right)$, Combined with Eq. (33), the $S_{e}\left(K, \tau_{0}\right)-M$ curve is obtained with a reference parameter $l_{0}$. Let $l_{0}=L \rightarrow 0$, the yielding process of interface can be evaluated.

It is obvious that, with the increase of bending moment, the bending rigidity of layered structure remains constant first and starts to reduce when bending moment reaches to a critical value, as the shear stress of interface climbs its critical shear resistance and yields. With the yield region grows, the rigidity of bilayer beam approaches to $S_{0}$, i.e. layered beam gradually become non-adhesion.

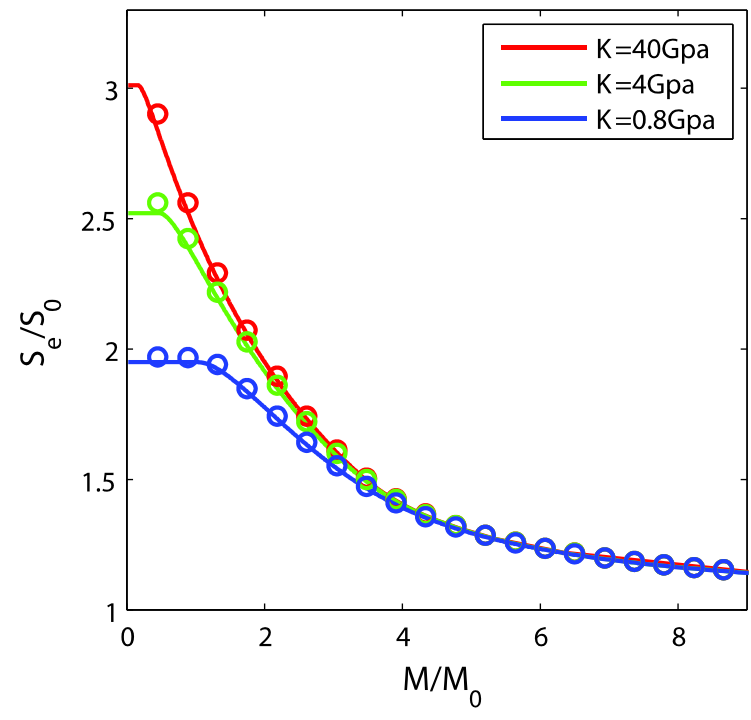

(a)

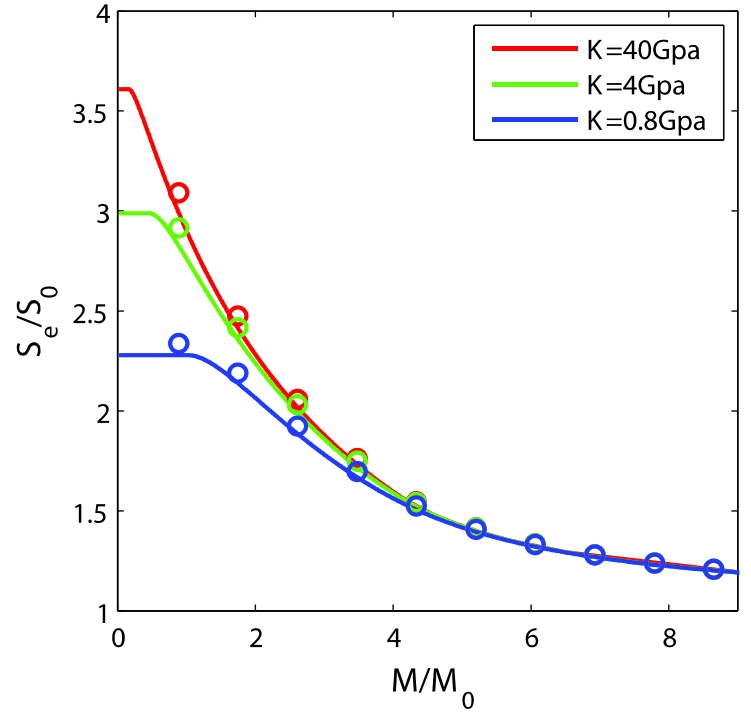

(b)

Fig. 5. Stiffness degradation with different interfacial rigidity: (a): the solid lines are the analytical results obtained by the model, and the dots correspond to FEM simulations (Case A). (b) Analytical and simulation results for the beam composed of identical layers (Case B). $S_{0}$ and $M_{0}$ are defined in Table 1 . 


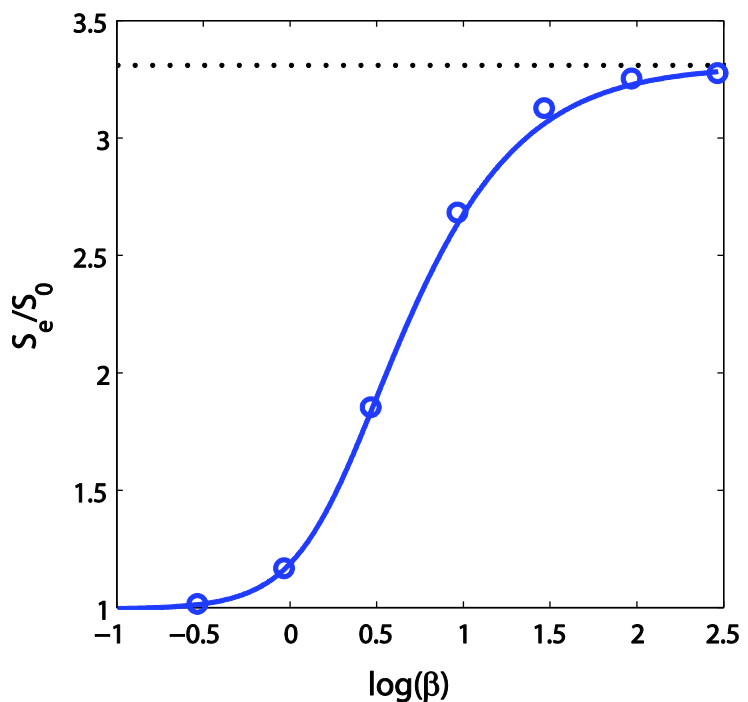

(a)

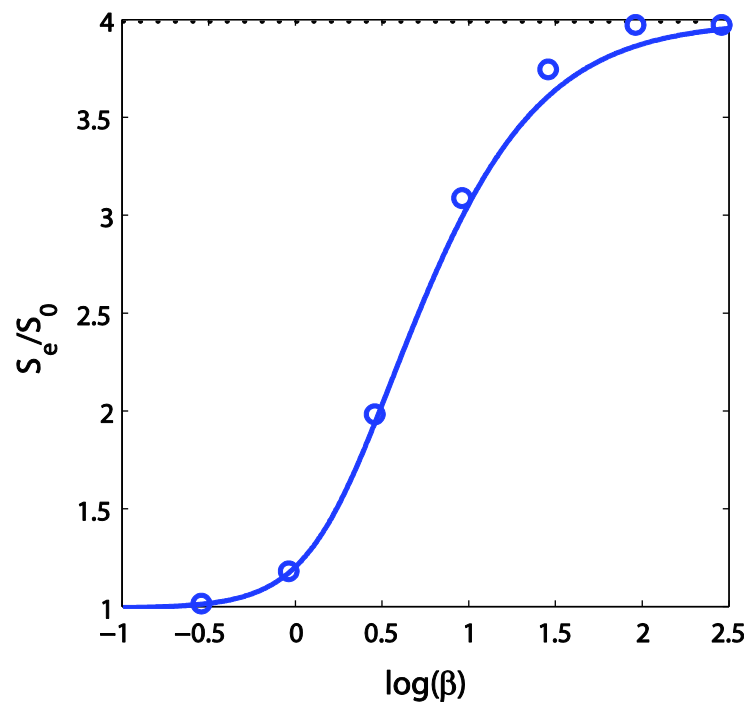

(b)

Fig. 6. Initial bending rigidity of layered beam as a function of $\beta$ (Eq. (27)). Simulation (cycles) and theoretical prediction (solid line) for the bending rigidity as a function of $\log (\beta)$ in a bilayer beam with dissimilar layers for (a). Case A; (b). Case B. Note that the bending rigidity of the perfectly bonded bilayer is four times of that of the unbonded bilayer.

As we can see from Fig. 5, the interfacial rigidity influences mostly on the initial stiffness of the bilayer beam. We know that, for case $\mathrm{B}$, the bending rigidity of the perfectly bonded bilayer is four times of that of the unbonded bilayer. For further investigation on the influence of interfacial stiffness, the defined non-dimensional parameter $\beta=A L$ (see Eq. (28)) is helpful.

Fig. 6 shows the relationship between initial stiffness of the layered beam and the parameter log $(\beta)$. For high $\beta$ values, bilayer beam behaves well combined with higher bending rigidity; for less $\beta$ values, bilayer beam can not bond tightly and thus bending rigidity drops. In this diagram, we see, $\beta$ is an essential parameter which directly decide how tightly the two layers are bonded through the interface. Now we investigate how the interfacial strength $\tau_{0}$ influences the bilayered beam's bending performance, without loss of generality, a particular interfacial stiffness $K=80 \mathrm{Gpa}$ is considered.

In Fig. 7, we present the rigidity degradation under the increasing load $M$ with a variety of interfacial stiffness for case $A$

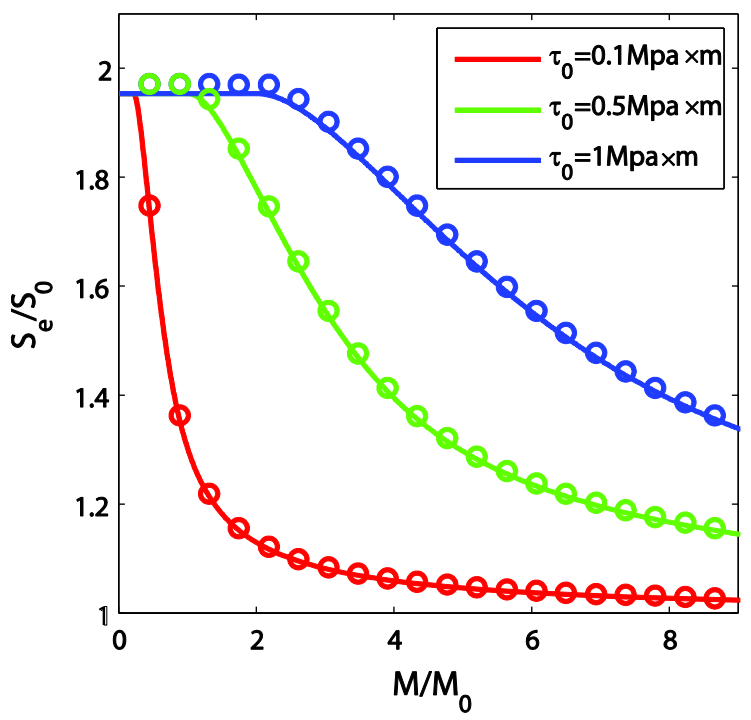

(a)

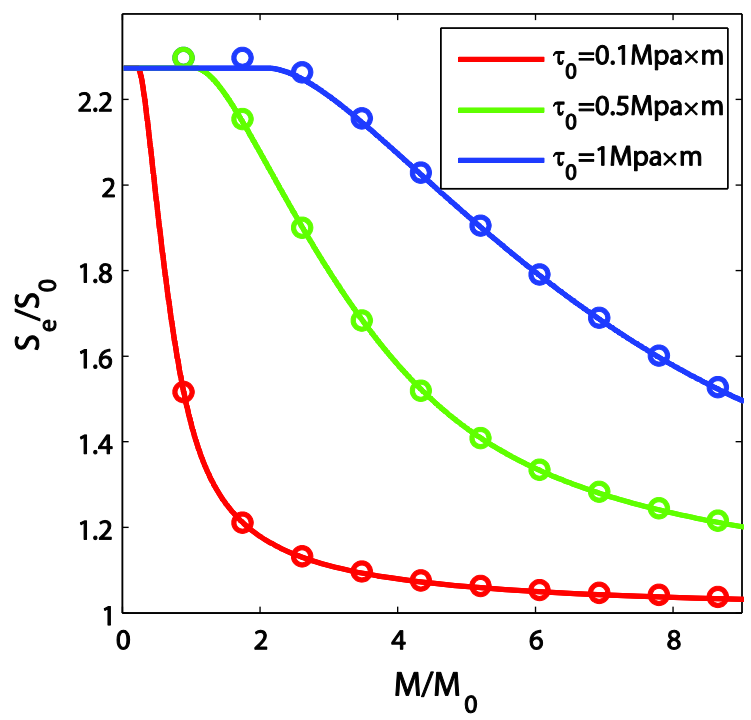

(b)

Fig. 7. Stiffness degradation with different interfacial strength. $\left(S_{e}=S_{e}\left(K, \tau_{0}, M\right)\right.$ with $\tau_{0}=0.5 \mathrm{Mpa} \mathrm{m}=$ const.). Simulation (cycles) and theoretical prediction (solid line) for the bilayer beam with dissimilar layers for (a) Case A; (b) Case B. The analytical results is obtained using the method for Fig. 5 with different values of $K$ and $\tau_{0}$. 


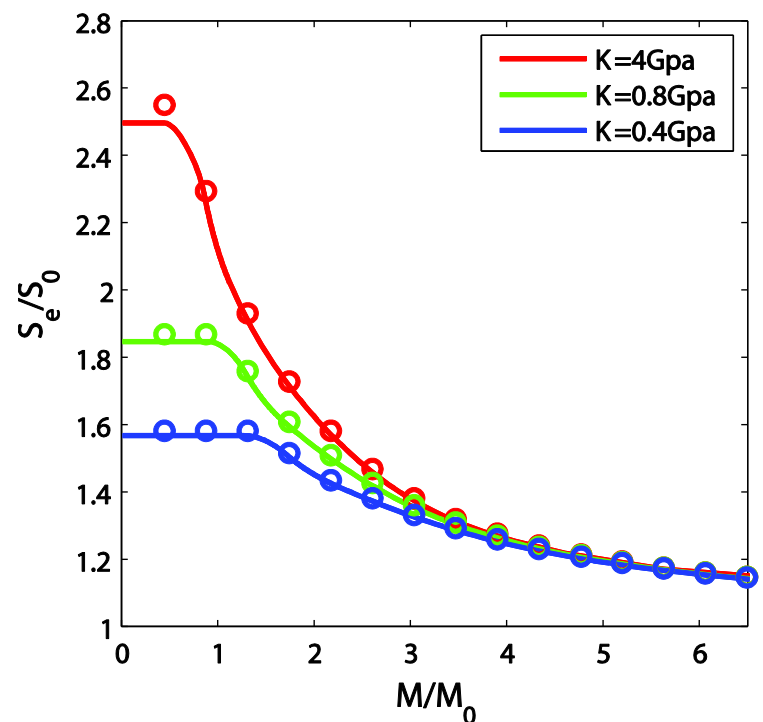

(a)

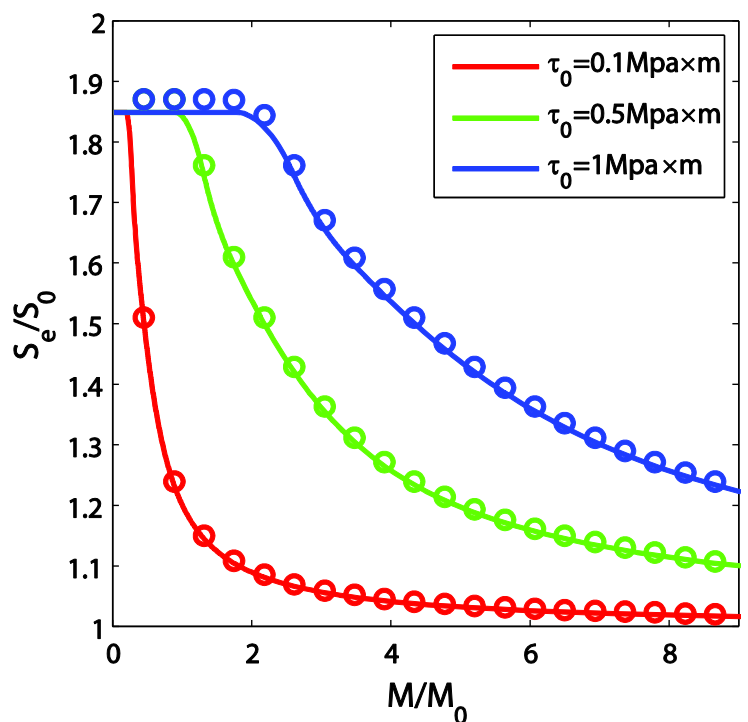

(b)

Fig. 8. Bending of layered structures with pre-existing interfacial failure. (a) $S_{e}=S_{e}\left(K, \tau_{0}, M\right)$ with $\tau_{0}=0.5 \mathrm{Mpa} \mathrm{m}=$ const. Simulation (cycles) and theoretical prediction (solid line). (b) $S_{e}=S_{e}\left(K, \tau_{0}, M\right)$ with $K=0.8 \mathrm{Gpa}=$ const. Simulation (cycles) and theoretical prediction (solid line).

and B. It shows that, higher interfacial strength leads to higher critical load; and most importantly, for higher interfacial shear resistance, the rigidity of bilayer beam reduces more slowly, that means a layered structure with less interfacial strength is more flexible than the one with higher interfacial strength.

\subsection{The pre-existing interfacial failure case}

From the semi-analytical solution derived in the previous section, we have $S_{e}=S_{e}\left(K, \tau_{0}, l_{s}\left(l_{e}\right), l_{a}, M\right)$. Now a bilayer beam with a pre-existing crack was investigated (see Fig. 4), the geometry of the crack is $l_{s}=0.6 L, l_{a}=0.2 \mathrm{~L}$ and the geometry of the beam follows the case $A$ in Section 4.2 (Table 1 ). Let $S_{0}, M_{0}$ be the values listed in Table 1 . Thus, with a fixed pre-existing

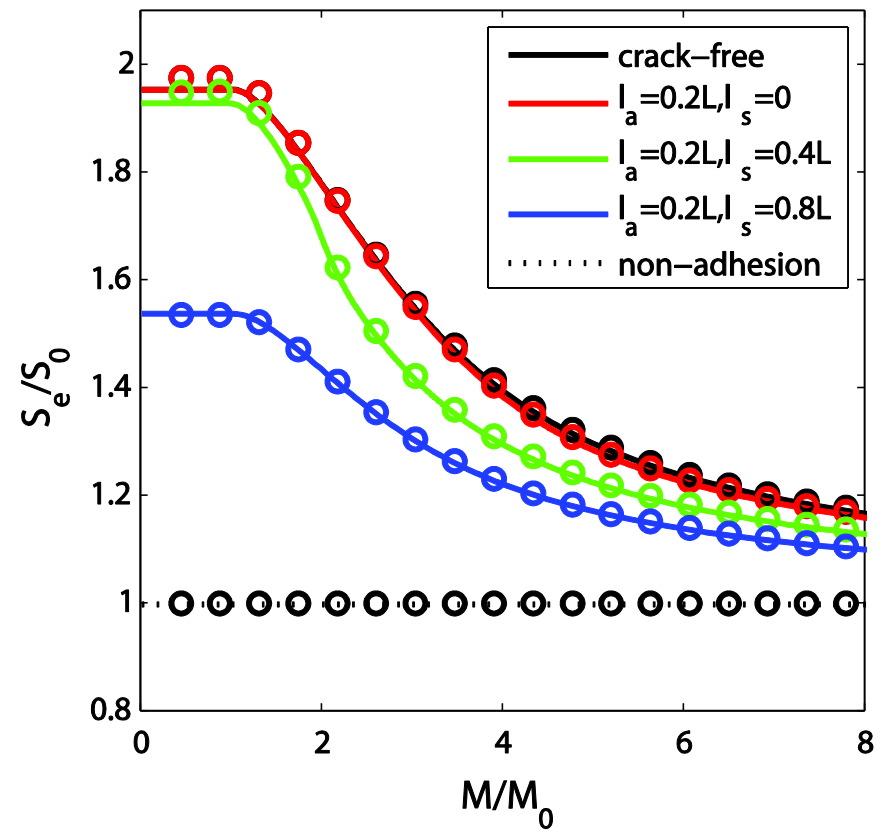

Fig. 9. Rigidity degradation with pre-existing interfacial failure, where we let $K=0.8 \mathrm{Gpa}$ and $\tau_{0}=0.5 \mathrm{Mpa} \mathrm{m}$. We consider cases when the crack is located at different positions. The solid black line corresponds to the crack-free case; the dashed black line is the non-adhesion result. Three different crack positions were investigated. Here $l_{s}, l_{a}$ are the geometrical characteristics of the crack, as seen in Fig. 4. 


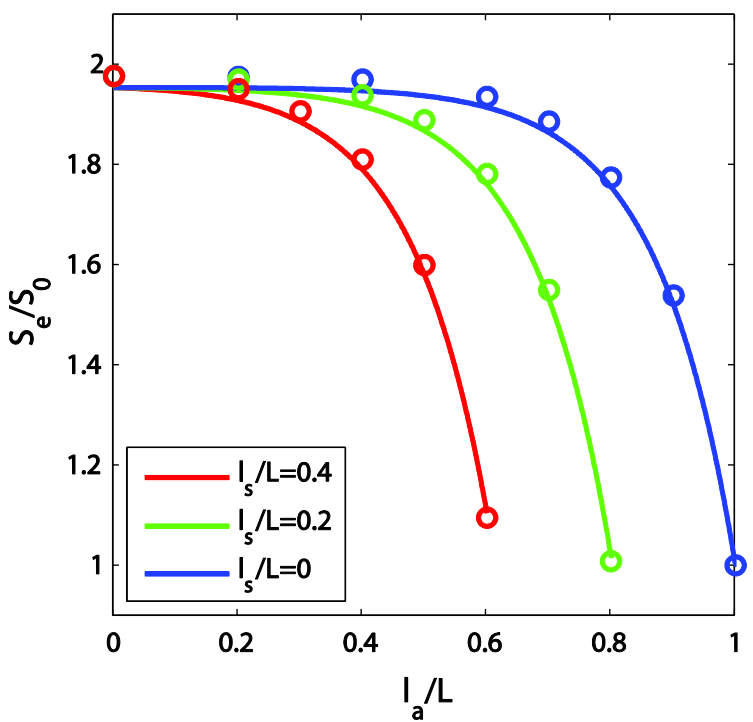

(a)

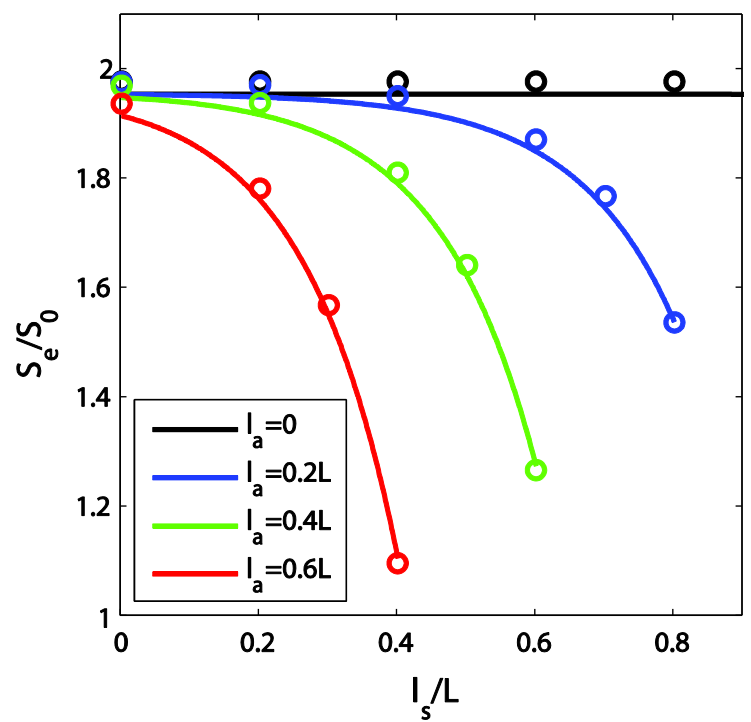

(b)

Fig. 10. Rigidity of layered beam with pre-existing crack: (a) For a fixed crack position $l_{s}$ (the left-hand side of crack), Rigidity of layered beam decreases as the crack length increases. (b) For a constant crack length $l_{a}$, the rigidity of layered beam decreases as the crack close to the free-end.

crack case, bending rigidity becomes $S_{e}=S_{e}\left(K, \tau_{0}, M\right)$. Using the same method above, bending behaviors with different interfacial property can be evaluated. Fig. 8 shows the same results as the previous crack-free structure. Here, the analytical results consist of four parts which are discussed in Section 3 separately. Solving the Eqs. (44), (50), (54) and (58), we have the deflection function for those four periods, and by definition $S_{e}=\frac{M}{\theta}=\frac{M}{w^{\prime}(L)}$, we obtain the $S_{e}-M$ curves for different $K$ and $\tau_{0}$.

The semi-analytical solution $S_{e}=S_{e}\left(K, \tau_{0}, l_{s}\left(l_{e}\right), l_{a}, M\right)$ indicates that the position of the pre-existing crack affects the bending rigidity also (Fig. 9).

The crack position influences the bending behavior as the figure shows, the beam with a pre-existing crack locating at the fixed end have little difference with the crack-free case, and with a pre-existing crack close to free end, the beam becomes more compliant. More importantly, the most difference between those curves is the initial bending rigidity. When only consider the elastic behavior, we may explore dependence of rigidity of the bilayer beam with pre-existing using deflection function in Section 3.2.1. With the deflection and using the definition of $S_{e}=\frac{M}{\theta}=\frac{M}{w^{\prime}(L)}$, we plot the relation between initial bending rigidity (without yielding) and the position or length of pre-existing failure, as seen in Fig. 10. The rigidity of the bilayer beam decreases as the crack region getting closer to the free-end of the beam.

\section{Circular bilayer plate with central interfacial crack}

In this section, we supply analytical solution to a more practical boundary value problem that a circular bilayer plate is subjected to central load. We are interested in how elastic interfacial deformation and pre-existing central circular crack would influence the bending properties of the plate. In Fig. 11, we show such a bilayer plate. At $r=R$, the plate is simply supported. The material properties and the geometry of the plate are shown in the illustration.

\subsection{Analytical formulation}

We are interested in quantifying the influence of interfacial deformation and pre-existing interfacial crack on the bending stiffness of the plate. Such change could be detected by non-destructive detecting techniques. Follow the steps

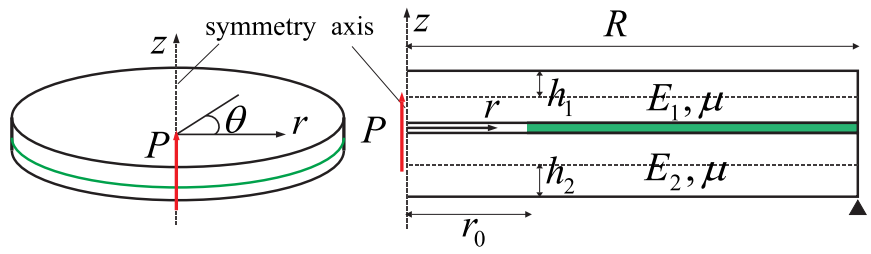

Fig. 11. geometry and properties of bilayer circular plate with pre-existing interfacial crack with radius $r_{0}$. The plate is simply supported at the edge, $r=R$. A central load $P$ is exerted at the center of the circular plate. 
described in Section 2, we assume the normal separation of the bilayer plate is negligible, thus the deflection of the upper and bottom layer are the same, i.e. $\mathrm{w}=\mathrm{w}(\mathrm{r})$. Under this assumption, we write the displacement field as below:

$$
u=\left\{\begin{array}{l}
u_{1}(r, z)=u_{10}(r)-\left(z-h_{1}\right) \frac{d w}{d r}, z \geq 0 \\
u_{2}(r, z)=u_{20}(x)-\left(z+h_{2}\right) \frac{d w}{d r}, z \leq 0
\end{array}\right.
$$

Again, subscripts ' 1 ' and '2' refer to the top and bottom layers, respectively. Now the strains along the radial direction are:

$$
\varepsilon_{r i}=\frac{\partial u_{\mathrm{i}}}{\partial r}, \varepsilon_{\theta i}=\frac{1}{r} u_{i}
$$

And the radial stresses are:

$$
\sigma_{r i}=\frac{E_{i}}{1-\mu^{2}}\left(\varepsilon_{r i}+\mu \varepsilon_{\theta i}\right), \sigma_{\theta i}=\frac{E_{i}}{1-\mu^{2}}\left(\varepsilon_{\theta i}+\mu \varepsilon_{r i}\right)
$$

The corresponding force and moment equations are given as:

$$
\begin{aligned}
& \left\{\begin{array}{l}
N_{r i}=\int \sigma_{r i} d z=D_{f i}\left(u_{i 0}^{\prime}+\frac{\mu}{r} u_{i 0}\right) \\
N_{\theta i}=\int \sigma_{\theta i} d z=D_{f i}\left(\mu u_{i 0}^{\prime}+\frac{1}{r} u_{i 0}\right)
\end{array}\right. \\
& \left\{\begin{array}{l}
M_{r i}=\int \sigma_{r i} z d z=-D_{M i}\left(w^{\prime \prime}+\frac{\mu}{r} w^{\prime}\right) \\
M_{\theta i}=\int \sigma_{\theta i} d z=-D_{M i}\left(\mu w^{\prime \prime}+\frac{1}{r} w^{\prime}\right)
\end{array}\right.
\end{aligned}
$$

with:

$$
D_{f i}=\frac{2 E_{i} h_{i}}{1-\mu^{2}}, D_{M i}=\frac{E_{i} h_{i}^{3}}{12\left(1-\mu^{2}\right)}
$$

where $\mu$ is the Poisson's ratio. For simplicity and without loss of generality, we assume that the Poisson's ratio of the two plates is the same.

From the free-body diagram given in Fig. 12, we obtain the equilibrium equations:

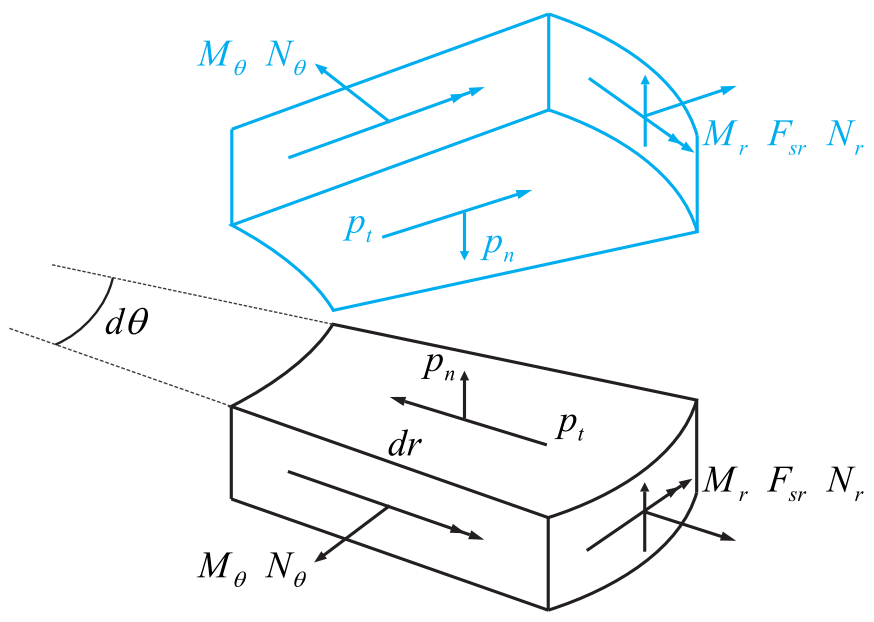

Fig. 12. Free-body diagram of an infinitesimal element. Here $M_{r}, M_{\theta}, N_{r}, N_{\theta}, F_{s r}$ stand for the inner forces per unit length of the plate, and $p_{t}$, $p_{n}$ are the tangential and normal stress of the interface respectively. 


$$
\begin{gathered}
N_{r i}^{\prime}+\frac{1}{r}\left(N_{r i}-N_{\theta i}\right)=\mp p_{t} \\
M_{r i}^{\prime}+\frac{1}{r}\left(M_{r i}-M_{\theta i}\right)=F_{s r i}+p_{t} \\
F_{s r 1}+F_{s r 2}=\frac{P}{2 \pi r}
\end{gathered}
$$

The displace jump across the interface is:

$$
\delta=u_{2}(r, 0)-u_{1}(r, 0)=u_{20}-u_{10}-\left(h_{1}+h_{2}\right) w^{\prime}
$$

Now the above equations could be rewritten as:

$$
\begin{aligned}
& \delta^{\prime \prime}+\frac{1}{r} \delta^{\prime}-\frac{1}{r^{2}} \delta=A^{2} \frac{p_{t}}{K}+C H \frac{1}{r} \\
& w^{\prime \prime \prime}+\frac{1}{r} w^{\prime \prime}-\frac{1}{r^{2}} w^{\prime}=B \frac{p_{t}}{K}+C \frac{1}{r}
\end{aligned}
$$

with

$$
\begin{gathered}
A^{2}=K\left(\frac{1}{D_{f 1}}+\frac{1}{D_{f 2}}+\frac{H^{2}}{D_{M 1}+D_{M 2}}\right) \\
B=K \frac{H}{D_{M 1}+D_{M 2}}, C=\frac{P}{2 \pi\left(D_{M 1}+D_{M 2}\right)}, H=h_{1}+h_{2}
\end{gathered}
$$

Now combining the constitutive relation of the interface:

$$
p_{t}=\left\{\begin{array}{l}
K \delta, \text { elastic region } \\
0, \text { crack region }
\end{array}\right.
$$

Noting that, the unit of $K$ is $\left[\mathrm{N} \mathrm{m}^{-3}\right]$ as the units of $p_{t}$ is $\left[\mathrm{N} \mathrm{m}^{-2}\right]$, as shows in Fig. 12. It is different from the definition in Section 2 due to the geometrical difference between rectangular bilayers and circular bilayers. We first solve Eq. (68) to obtain the displace jump, and then substitute the result to Eq. (69) to solve the deflection of the bilayer plate.

The general solution of Eq. (68) can be written as

$$
\delta(r)=\left\{\begin{array}{l}
A_{1} I_{1}(A r)+A_{2} K_{1}(A r)-\frac{C H}{A^{2} r}, \text { elastic } \\
C_{1} r+\frac{C_{2}}{r}+\frac{1}{2} C H r \ln r, \text { crack }
\end{array}\right.
$$

Hence the general solution of Eq. (69) is given as

$$
w(r)=\left\{\begin{array}{l}
A_{0}+A_{3} \ln r+A_{4} r^{2}+(1-k) \frac{C}{4} r^{2} \ln r+\frac{B}{A^{3}}\left[A_{1} I_{0}(A r)-A_{2} K_{0}(A r)\right], \text { elastic } \\
C_{0}+C_{3} \ln r+C_{4} r^{2}+\frac{C}{4} r^{2} \ln r, \mathrm{crack}
\end{array}\right.
$$

Here $I_{0}, I_{1}$ and $K_{0}, K_{1}$ are the modified Bessel function of the first and the second kind, respectively. The constants $A_{0}$, $A_{1}$, $A_{2}, A_{3}$, and $A_{4}$ for the elastic interface, and the additional $C_{0}, C_{1}, C_{2}, C_{3}$, and $C_{4}$ for the cracked interface in Eqs. (72) and (73) can be determined by the boundary conditions. In our case the boundary conditions are listed below (in terms of $\delta$ and $w$ ):

$$
\begin{aligned}
& \left\{\begin{array}{l}
\delta(0)=0 \\
\delta^{\prime}(R)+\frac{\mu}{r} \delta(R)=0
\end{array}\right. \\
& \left\{\begin{array}{l}
w(0) \neq \infty \\
w^{\prime}(R)+\frac{\mu}{r} w^{\prime}(R)=0 \\
w(L)=0
\end{array}\right.
\end{aligned}
$$

Additional continuous conditions may be needed when there is a pre-existing crack in the interface. The values include $\delta\left(r_{0}\right), \delta^{\prime}\left(r_{0}\right)+\frac{\mu}{r} \delta\left(r_{0}\right), w\left(r_{0}\right), w^{\prime}\left(r_{0}\right), w^{\prime \prime}\left(r_{0}\right)+\frac{\mu}{r} w^{\prime}\left(r_{0}\right)$. With the equations shown above, we may obtain the deflection of the bilayer axisymmetric circular plate. 


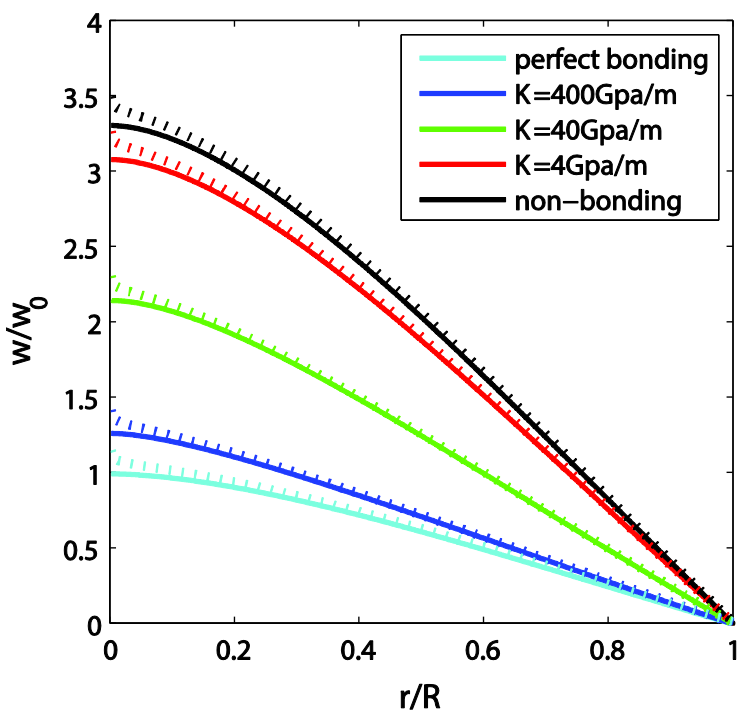

(a)

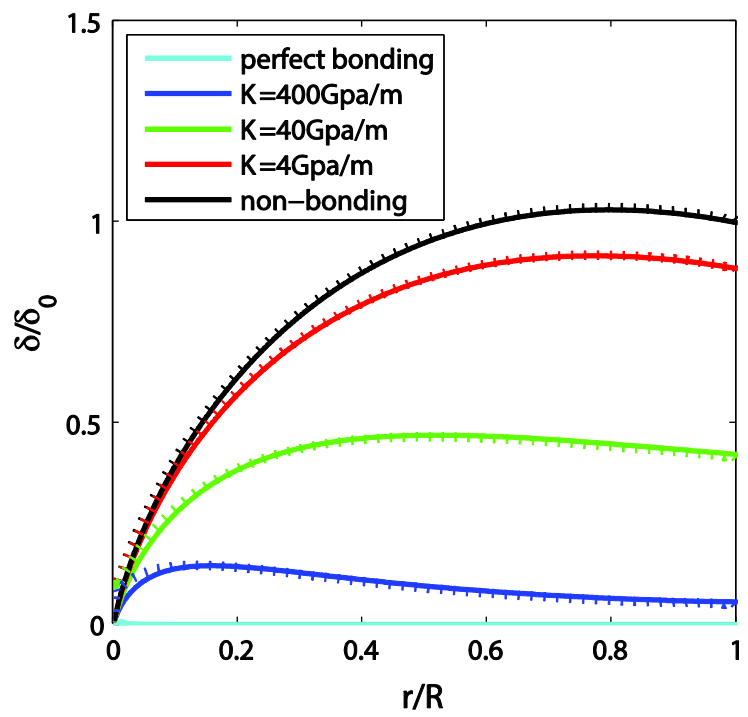

(b)

Fig. 13. Deformation of the bilayer plate with elastic interface. The solid lines and dashed lines are theoretical predictions and results from finite-element simulations, respectively. (a) Deflection of the plate for different interfacial rigidity. (b) The shear displacement across the interface for different interfacial rigidity. Here $w_{0}$ is the theoretical deflection in the center of a bilayer plate with perfectly bonded interface, and $\delta_{0}$ is the theoretical displace jump in the boundary of a bilayer plate with free interface sliding.

\subsection{Numerical verification}

Instead of writing out the long explicit expression of all ten constants $A_{0}$ to $A_{4}$ and $C_{0}$ to $C_{4}$, we use Matlab to solve the above equations and get the theoretical solution. We further performed finite element simulations to validate our theoretical results. We consider a bilayer circular plate with material properties $E_{1}=60 \mathrm{GPa}, E_{2}=80 \mathrm{GPa}$ and $\mu=0$. 3. The geometries are $h_{1}=0.50 \mathrm{~cm}, h_{2}=0.25 \mathrm{~cm}$, and $R=10 \mathrm{~cm}$. We apply a concentrated load of $P=1 \mathrm{kN}$ at the center of the circular plate.

\subsubsection{Crack-free elastic interface}

In Fig. 13a, we first show the deformation of the bilayer plate with elastic interface. It is noted that the interfacial stiffness significantly alters the bending rigidity of the circular plate: the larger value of interfacial rigidity, the smaller deflection at

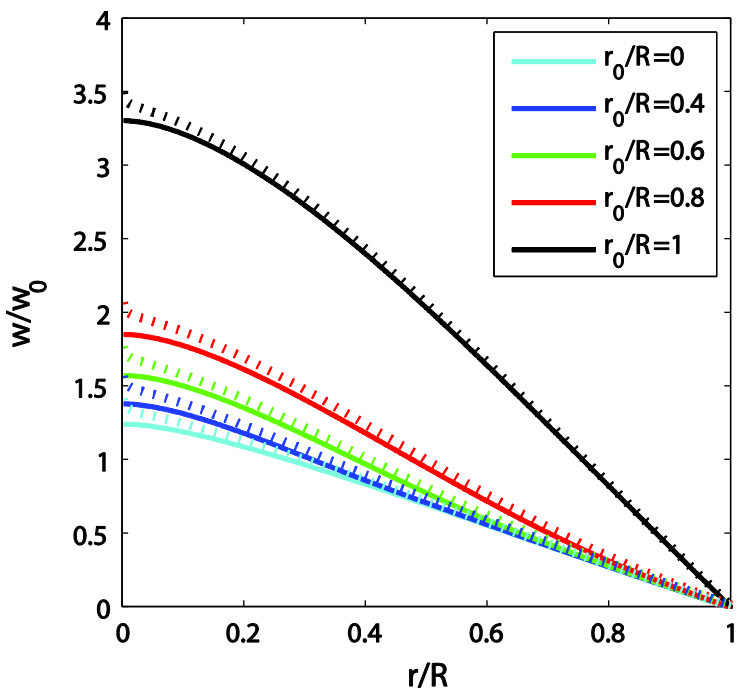

(a)

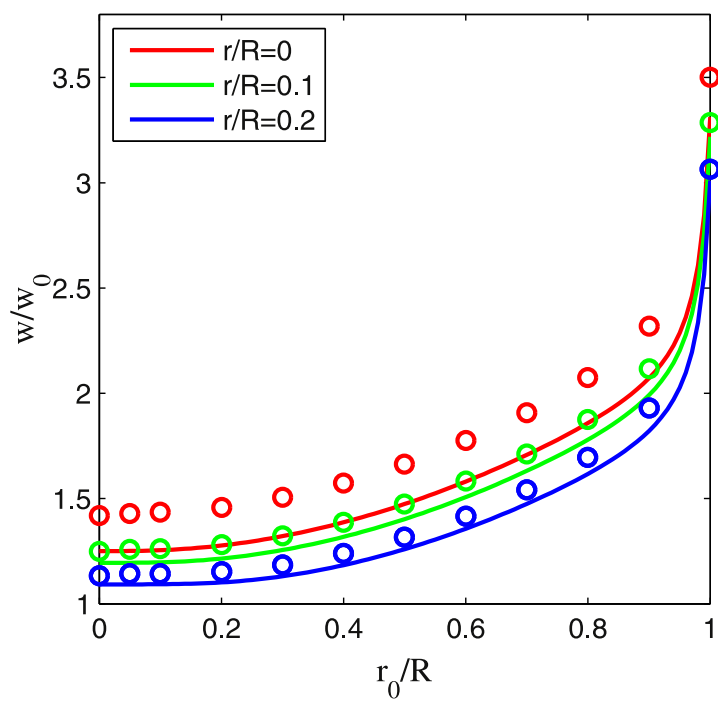

(b)

Fig. 14. Deformation of the bilayer plate with pre-existing crack in the interface. (a) Deflection curves for plates of different central crack radius. (b) The deflection of the plate in position $r / R=0, r / R=0.1$ and $r / R=0.2$ as a function of the crack radius. 
the central of the plate. The difference in bending stiffness is slightly less than four, due to the geometrical constraints. There is a very good match between theoretical prediction and numerical results from finite element simulations. Unlike those rectangular beams where maximum interfacial shear stress first occurs at the end of the beam, we see that the critical point where the interfacial stress maximizes is a function of the interfacial rigidity, as clearly seen in Fig. $13 \mathrm{~b}$.

\subsubsection{Interface with pre-existing crack}

Now we consider the case of pre-existing crack in the center of the circular plate. We now use a constant interfacial rigidity of $K=400 \mathrm{GPa} / \mathrm{m}$ for discussion. Fig. 14 shows how the change in crack size would alter the bending behavior of the plate. We first show the deflections of the circular plates with central crack of different radii in Fig. 14a. Again the theoretical predictions match well with numerical results from finite element simulations. Under a constant concentrated load, the deflection of the plate increases with larger crack radius, as seen in Fig. 14b. There is a singularity at the center of the plate when a concentrated force is applied here. Hence the results closing to that point are less trust-worthy. We adopt the deflection at different points, $r / R=0, r / R=0.1$ and $r / R=0.2$. It is clearly seen that if we choose a point away from the center, the theoretical prediction and the numerical result match well.

\section{Discussions and conclusions}

In response to the global pressure to reduce $\mathrm{CO}_{2}$ emission, major energy consumers like transportation industry are pushing the limit of energy saving strategies. Among those endeavors, light-weight composite materials and structures with rich interfaces are broadly employed for better combination of reliability and functionality. The interfacial properties are hence deterministic to the overall mechanical behavior of the structures and are crucial for safety. In particular, how to identify the interfacial integrity using non-destructive techniques is a topic of broad interest. Here we focus on revealing the influence of interfacial shearing or pre-existing cracks on the bending stiffness of layered structures. We formulate the analytical solution of the bending rigidity of bilayered structures as a function of the interfacial shear strength, and also give a semi-analytical solution to the bending of bilayers when both shearing and pre-existing crack presents in the interface of the bilayers. The analytical solutions were validated by finite element simulations. While the critical bending stiffness when the interface starts to slide plastically is proportional to the interfacial shear strength, there is a strong nonlinearity between the rigidity and the applied bending after interfacial plastic shearing. One may use the linear relationship between the critical bending stiffness to trigger plastic shearing and the shear strength (Eq. (34)) to measure the shear strength of interfaces.

In addition, we expect that the analysis given here could be used to understand the significant stiffness difference in novel structures and materials. For example, as the perfect bonding case and the unbonded case in the interface of a bilayer would lead to a maximum difference by a factor of 4 (Fig. 6b), the significant difference in bending stiffness between a monolayer graphene and a bi-layer graphene (Koening et al., 2011) cannot be fully addressed by interfacial bonding. There must be dramatic difference in terms of electron distribution in the out-of-plane direction between the monolayer and the bi-layer graphene. The analysis can also be used for non-destructive detection of interfacial crack when stiffness (typically resulted in change in vibration frequency) can be probed. In addition, the abrupt change of the bending stiffness in response to pre-existing cracks in the interface of both rectangular bilayers (see Fig. 10) and circular bilayers (see Fig. 14) could be utilized to identify the size and the location of possible cracks in interfaces. The latter is particular meaningful for composite shell structures which are now broadly used in aircrafts.

\section{Acknowledgments}

The authors acknowledge support from the National Natural Science Foundation of China (NSFC) (Grant no. 11425211), and MOST 973 of China (Grant no. 2012CB937500).

\section{References}

Adekola, A.O., 1968. Partial interaction between elastically connected elements of a composite beam. Int. J. Solids Struct. 4, 1125-1135. Ayoub, A., Filippou, F.C., 2000. Mixed formulation of nonlinear steel-concrete composite beam element. J. Struct. Eng. (ASCE) 126 (3), $371-381$. Boal, D., 2002. Mechanics of the Cell. Cambridge University Press.

Camacho, G.T., Ortiz, M., 1996. Computational modeling of impact damage in brittle materials. Int. J. Solids Struct. 33, $2899-2938$.

Cas, B., Saje, M., Planinc, I., 2004. Non-linear finite element analysis of composite planar frames with an interlayer slip. Comput. Struct. 82, $1901-1912$.

Dugdale, D.S., 1960. Yielding of steel sheets containing slits. J. Mech. Phys. Solids 8, 100-104.

Gao, H.J., Klein, P.A., 1998. Numerical simulation of crack growth in an isotropic solid with randomized internal cohesive bonds. J. Mech. Phys. Solids 46, $187-218$.

Gara, F., Ranzi, G., Leoni, G., 2006. Displacement-based formulations for composite beams with longitudinal slip and vertical uplift. Int. J. Numer. Methods Eng. 65, 1197-1220.

Girhammar, U.A., Gopu, V.K.A., 1993. Composite beam-columns with interlayer slip- exact analysis. J. Struct. Eng. 119, $1265-1282$.

Girhammar, U.A., Pan, D.H., 2007. Exact static analysis of partially composite beams and beam-columns. Int. J. Mech. Sci. 49, 239-255.

Hutchinson, J.W., Evans, A.G., 2000. Mechanics of materials: top-down approaches to fracture. Acta Mater. 48, 125-135. 
Koening, S.P., Boddeti, N.G., Dunn, M.L., Bunch, J.S., 2011. Ultrastrong adhesion of graphene membranes. Nat. Nanotechnol. 6, 543-546.

Lu, Q., Arroyo, M., Huang, R.J., 2009. Phys. D: Appl. Phys. 42, 102002.

McIntosh, T.J., Simon, S.A., 2006. Roles of bilayer material properties in function and distribution of membrane proteins. Annu. Rev. Biophys. Biomol. Struct. 35, 177-198.

Newmark, N.M., Siess, C.P., Viest, I.M., 1951. Tests and analysis of composite beams with incomplete interaction. Proc. Soc. Exp. Stress Anal. 9, 75-92. Nguyen, Q.H., Martinelli, E., Hjiaj, M., 2011. Derivation of the exact stiffness matrix for a two-layer Timoshenko beam element with patial interaction. Eng. Struct. 33, 298-307.

Shen, Y.K., Wu, H.A., 2012. Interlayer shear effect on multilayer graphene subjected to bending. Appl. Phys. Lett. 100, 101909.

Schnabl, S., Saje, M., Turk, G., Planinc, I., 2007. Analytical solution of two-layer beam taking into account interlayer slip and shear deformation. J. Struct. Eng. 133, 886-894.

Salari, M.R., Spacone, E., 2001. Analysis of steel-concrete composite frames with bond-slip. J Struct. Eng. ASCE 127, 1243-1250.

Wei, Y.J., 2014. A stochastic description on the traction-separation law of an interface with non-covalent bonding. J. Mech. Phys. Solids 70, 227-241.

Wei, Y.J., Wang, B.L., Wu, J.T., Yang, R.G., Dunn, M.L., 2013. Bending rigidity and gaussian bending stiffness of single-layered graphene. Nano Lett. 13, 26-30. Xu, X.P., Needleman, A., 1994. Numerical simulations of fast crack growth in brittle solids. J. Mech. Phys. Solids 42, 1397-1434.

Xu, R., Wu, Y., 2007. Static, dynamic and buckling analysis of partial interaction composite members using Timoshenko's beam theory. Int. J. Mech. Sci. 49, 1139-1155. 